\title{
Vibratory monitoring of a spalling bearing defect in variable speed regime
}

\author{
Khalid Ait Sghir ${ }^{1, a}$, Fabrice Bolaers ${ }^{1}$, Olivier Cousinard ${ }^{1,2}$ and Jean-Paul Dron ${ }^{1}$ \\ ${ }^{1}$ Groupe de Recherche en Science Pour l'Ingénieur, Université de Reims Champagne Ardenne, UFR Sciences Exactes \\ et Naturelles ; Moulin de la Housse, BP 1039, 51687 Reims Cedex, France \\ 2 Société Altéad Industrie Est, 11 rue du colonel Charbonneaux, 51100 Reims, France
}

Received 14 December 2012, Accepted 24 April 2013

\begin{abstract}
Rotating machines monitoring in a non-stationary regime, characterized by the operating parameters (speed, load) variation, presents a particular challenge. The speed variation has an impact on the vibratory response given by the accelerometers and therefore masks any defect that may be detected by classical indicators (for example $R M S$ value). To overcome this problem, a new indicator is proposed. For this, a signal accelerometer and a signal from an optical encoder are acquired simultaneously. An algorithm to estimate the instantaneous speed from the signal delivered by the optical encoder is applied. Then, each sample of the accelerometer signal is divided by its corresponding instantaneous speed sample. The $R M S$ value is then applied to the resulting signal. A model simulation signal is used to test the proposed method. A test rig is performed to extract signals of different degradation states of thrust bearings in variable speed regime. The results show a correlation between the proposed $R M S$ value and the thrust bearings state in variable regime.
\end{abstract}

Key words: Rolling fatigue contact / spalling / thrust roller bearing / monitoring / vibratory analysis / instantaneous speed

\section{Nomenclature}

\begin{tabular}{|ll|}
\hline$A_{i}$ & $\begin{array}{l}\text { Amplitude modulation of the } i \text { th impact } \\
\text { of the simulate signal }\end{array}$ \\
$b(n)$ & Gaussian white noise \\
$C_{1}$ & Variable which depends to the bearing geometry \\
$C_{2}$ & Coefficient which is related to $h(n)$ \\
$d e f$ & Defect size \\
$E_{i}(n)$ & Sequence of the impact forces \\
$F$ & Rotating frequency \\
$F_{i}$ & Impact force \\
$h(n)$ & Impulse response \\
$Q$ & Load applied to the bearing \\
$R M S$ & Root mean square value \\
$R M S_{0}$ & RMS in the free fault case \\
$T$ & Time between two impacts \\
$v_{i}$ & Rotating speed of the inner race \\
\hline
\end{tabular}

\section{Introduction}

In industrial applications, rolling element bearings are present in the majority of the rotating machines and are

\footnotetext{
${ }^{a}$ Corresponding author: khalid.ait-sghir@univ-reims.fr
}

considered among the most critical components. A defect in a bearing, unless detected in time, causes malfunction and may even lead to catastrophic failure of the machinery. The dominant mode of failure of rolling element bearings is spalling of the races or the rolling elements, caused when a fatigue crack begins below the surface of the metal and propagates towards the surface until a piece of metal breaks away to leave a small pit or spall [1]. It is important to detect such localized defect at its incipient stage in order to prevent long-term breakdowns or in some cases possible catastrophic failures.

Different methods are used for detection and diagnosis of bearing defects: vibrations, acoustic emissions, temperature measurements etc. Among these, the vibration analysis is the most broadly used. For the reason that in the presence of a defect, there is a significant increase in the vibration level and combined with a suitable signal processing method, a robust indicator may be suggested to incipient fault detection.

Under steady-state regime (load and speed constant), number of indicators have been proposed to monitoring the bearing state. The most used are the classical indicators; we find time indicators such as $R M S$ value [2], kurtosis [3], spectral indicators [4,5]. These classical indicators suppose that the vibration signals are stationary. Envelope detection [6] is an important signal processing 
technique that has been used extensively and its success has been demonstrated [7,8]. Time-frequency analysis, which is an effective tool for analyzing the behavior of non-stationary signals, is recently widely used [9] in steady regime. In recent years, the cyclostationary tools, which model the vibration signal as cyclostationary process, are proposed and have been efficient for the bearing fault detection $[10,11]$.

However, the bearing defects monitoring is more complicated when the operating parameters of systems are variables especially for the speed and for the load. This kind of operating regime is prevalent in industry, for example the tramway, plane motors, wind turbine etc. Under these non-stationary operations, the amplitude and frequency components are always changing because of speed variation (or load variation). This affects clearly the classical diagnostic tools, which are not suitable for non-stationary analysis, and therefore a potential fault can be masked. The proposed method must able to detect the fault in a variable regime. In the literature, several methods attempt to manage these issues in condition monitoring approach. In reference [12], a method is proposed based on time-frequency analysis techniques combined with the automatic feature extraction method and fuzzy inference in order to automatically diagnose bearing faults in a variable speed regime. In reference [13], it is found that the classical spectral indicator is linear with the operating conditions indicators. This linearity forms a slope which is used as parameter for monitoring the planetary gearbox used in bucket wheel excavators. In reference [14] a statistical approach is given in the case of variable speed, the signal is divided into segments where speed is considered as constant then the mean and covariance matrices for the features vector are calculated and the interpolation is made by the ranges of speed bins. A classification by a statistical approach is then applied to the diagnosis of a gearbox.

In this work, a method is proposed to eliminate the effect of speed variation on the classical indicator $R M S$ and to diagnose with the new $R M S$ value. The paper is organized as follows: Section 2 presents the proposed method. Section 3 presents the application to the simulate signals and Section 4 the experiments carried out. In Section 5 the result analyzes are given before finishing by a conclusion.

\section{The proposed method}

When the rolling element strikes a local defect (see Fig. 1a) a shock is introduced that excites high frequency resonances of the whole structure between the bearing and the response transducer. This shock caused by the impact force of the bearing element on the defect is periodic with $T$ and its shape is a Dirac with finite power (Fig. 1b). This Dirac is a resultant of the triangle, rectangle or parabolic form [15]. Several research works have developed analytical model of the impact force. For example a dynamic model is developed in [16] which gives the relation between the impact force magnitude and a linear

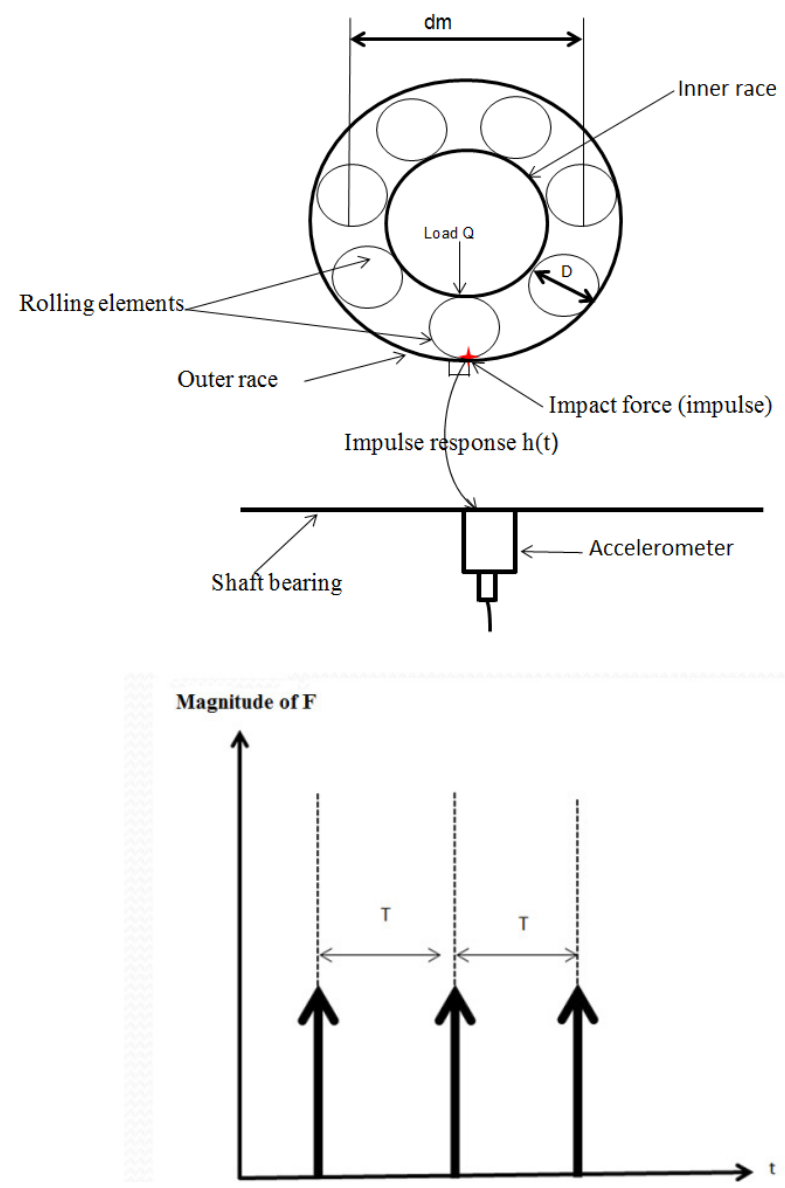

(a)

Fig. 1. (a) Modeling shock impact of the rolling element on a spalling defect, (b) Sequence of impact forces.

function of the rotational speed of inner race, the bearing geometry and the defect size. In this work, the model of the impact force $F_{i}$ established in [17] is considered and is presented by the following equation:

$$
F_{i}=C_{1} v_{i} Q d e f
$$

where $C_{1}$ is a variable that depends on the bearing geometry [17].

$v_{i}$ is the rotating speed of the inner race $(\mathrm{rpm})$

$Q$ is the load applied to the bearing (N)

def is the defect size $(\mathrm{mm})$

That force impact increases the level of vibration measured. In the other hand, the $R M S$ value is a widely used indicator that measures the level of static redressed energy of a vibration signal. Its estimation formula for a vibration signal is given by:

$$
R M S=\sqrt{\sum_{n=1}^{N} \frac{(x(n))^{2}}{N}}
$$

Where $N$ is the number of samples and $x(n)$ is the sample of vibration signal at time $n$.

The vibration signal $x(n)$ given by the acceleration sensor is modeled by the convolution product between 
the impact force $F_{i}(n)$ and the impulse response $h(n)$ of the system formed by the rolling element-outer raceshaft (Fig. 1a). This system is assumed to be linear timeinvariant. It is also assumed that the dynamic behavior of the machine does not contain resonances that may amplify vibration measurements. The signal $x(n)$ can be written as:

$$
x(n)=E_{i}(n) \otimes h(n)+b(n)
$$

With $E_{i}(n)=\sum_{k=-\infty}^{\infty} F_{i}^{k} \delta(n-k T)$ a sequence of the impact forces, $b(n)$ Gaussian white noise which represents the other vibration sources and $\otimes$ the convolution operation.

For a number of samples $N$, on which the $R M S$ value is computed, it is assumed that the amplitudes of the impulses $E_{i}(n)$ are constant. The convolution equation can be written as:

$$
x(n)=F_{i} \sum_{k=-\infty}^{\infty} h(n-k T)+b(n)
$$

The $R M S$ equation of the signal $x(n)$ is given by:

$$
R M S=\sqrt{\sum_{n=1}^{N} \frac{\left(F_{i} \sum_{k=-\infty}^{\infty} h(n-k T)\right)^{2}}{N}}+R M S_{0}
$$

where $R M S_{0}$ is the $R M S$ value of $b(n)$ (the $R M S$ in the free fault case).

Let $C_{2}=\sqrt{\sum_{n=1}^{N} \frac{\sum_{k=-\infty}^{\infty} h(n-k T)^{2}}{N}}$, a coefficient which is related to the impulse response $h(n)$ and which depends only on the system properties, the transmission path between the point of impact and the accelerometer sensor. The value of $R M S$ is then:

$$
R M S=F_{i} C_{2}+R M S_{0}=C_{1} C_{2} Q v_{i} d e f+R M S_{0}
$$

Equation (6) provides that the $R M S$ value of the signal $x(n)$ depends linearly on: the load $Q$, the coefficient $C_{1}$ which depends to the bearing geometry, the coefficient $C_{2}$ which depends to the impulse response $h(n)$, the size of defect def and the speed rotation of the inner race $v_{i}$.

The proposed method consists in eliminating the influence of speed rotation of the inner race $v_{i}$ from equation (6) and then in using the new $R M S$ value for the diagnosis. That is equivalent in equation (4), to divide the vibration signal at time $n$ by the corresponding instantaneous speed rotation of the inner race. The following algorithm, presented in Figure 2, allows us to:

- Simultaneously acquire the vibration signal $x(n)$ and the optical encoder signal $p(n)$;

- Estimate the instantaneous speed from the optical encoder and a smoothing is applied;

- Divide of the signal vibration by the instantaneous speed estimated;

- Calculate the $R M S$ value from the resulting signal and diagnose with the new $R M S$ value.

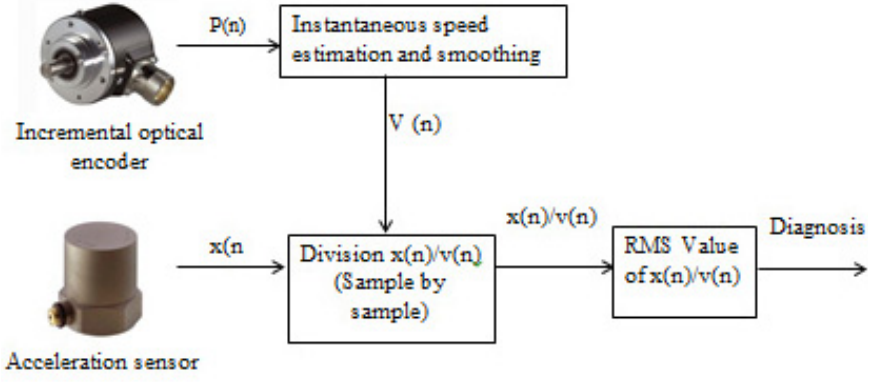

Fig. 2. Algorithm of the proposed indicator.

\section{Application to the synthetic signals}

In order to test the proposed method, a simple model of a vibration signal produced by a single defect is considered. The vibration signal model is written as:

$$
x(n)=\sum_{i} A_{i} h\left(n-i T-\tau_{i}\right)+b(n)
$$

Where $b(n)$ is an additive background noise which takes into account the effects of the other vibrations of the system, $h(n)$ is the impulse response generated by a single impact (it is assumed that the impulse response $h(n)$ is identical whatever the shape of the impact), $T$ is the time between two impacts (in a variable regime, $T=1 / F$ is linked to rotating frequency $F$ ), $A_{i}$ is the amplitudemodulation of the $i$ th impact force and $\tau_{i}$ is the time lag from its time shock $i T$ due to the presence of slip. This model has been successfully used to describe incipient faults in rolling element bearings $[18,19]$.

For the simulate signals three cases are considered: free fault, defect 1 and defect 2 . In the case of defect 2 , the modulation amplitude $A_{i}$ is more important than in defect 1 where in the free fault case is equal to zero. The sampling frequency is $10 \mathrm{kHz}$, the time of each signal is $90 \mathrm{~s}$ which gives $9 \times 10^{5}$ samples. The rotation speed variation presented in Figure $3 \mathrm{a}$ is considered. Figure $3 \mathrm{~b}$ presents the signals produced by the model (7) in the case of free fault and Figure $3 c$ in the presence of defect 2. It can be seen in these figures that simulate signals are modulated by the rotation speed variation. In the faulty case, it can be seen that the amplitude of signal is more important due to the presence of the defect.

The simulation signals are subdivided in segments (5000 points) and for each segment the $R M S$ value is computed and presented in Figure 4a. It can be seen in this figure that the speed variation has an impact on the $R M S$ values. For example the $R M S=1.2$ can indicates both free fault and faulty case.

Figure 4b presents the $R M S$ values after division the simulation signals by the corresponding instantaneous speeds. From this figure, one can see that the new $R M S$ value of the free fault is constant for all segments. When the fault is present, the new $R M S$ value begins rising and it can distinguish the faulty case from the free fault case whatever the speed variation. That increase of $R M S$ value is due to the presence of the impulse response generated by the impacts. 


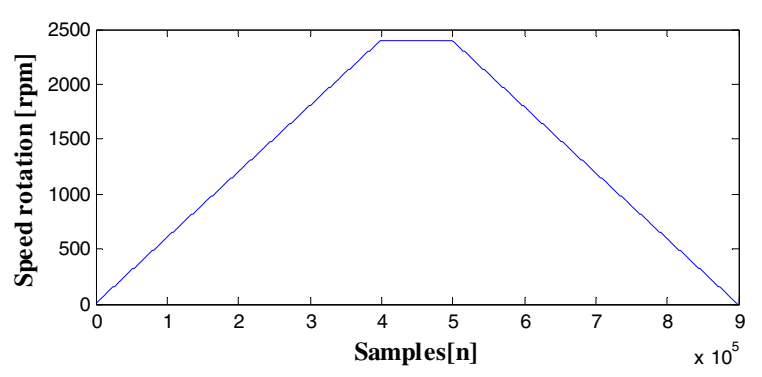

Free fault case

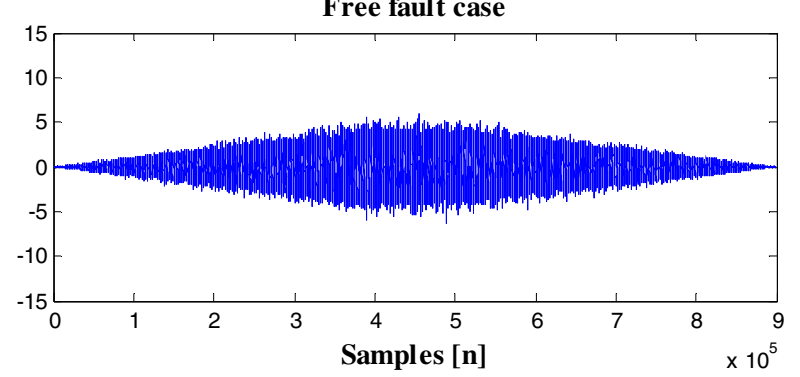

(b)

With defect 2

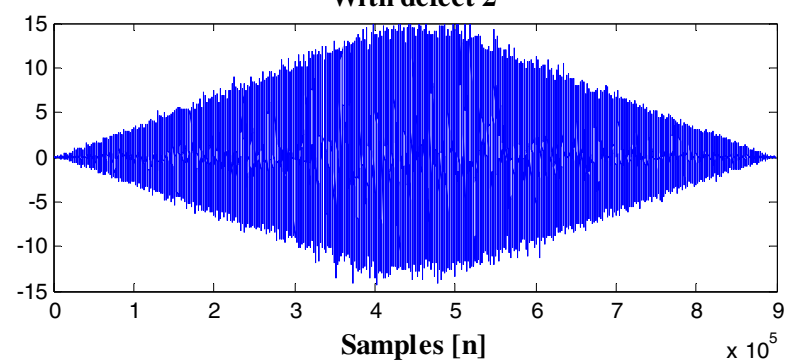

(c)

Fig. 3. (a) The speed rotation used, (b) Typical signal in the free fault case 1 (c) Typical signal in the presence of defect 2 .

\section{Test rig}

To test the proposed indicator on the real signals, a series of experiments is performed to extract signals, in variable regime, on a test bench (Fig. 5a). This bench is equipped with a brushless motor with $10 \mathrm{~kW}$ power driven by an inverter, a module for the thrust bearings fatigue, a device for applying a load on the thrust bearings and an oil injector for the lubrication. A ICP accelerometer sensor with sensitivity $9.87 \mathrm{mv} \cdot \mathrm{g}^{-1}$ is located on the axial direction of the thrust bearing. An incremental optical encoder, integrated into the motor, delivers $1024 \mathrm{point} /$ revolution. The optical encoder which is a position sensor allows us to estimate the instantaneous speed. For each test the signals from the accelerometer and the optical encoder are acquired simultaneously by a dynamic system acquisition. For the accelerometer signal the sampling frequency is $25600 \mathrm{~Hz}$ and for the optical encoder the sampling frequency is $102400 \mathrm{~Hz}$. The acquisition time is $35 \mathrm{~s}$ (or 896000 samples for the vibration signal). Figure 6 shows the variation cycle of the machine speed for all acquisitions: the speed rise area which is from $50 \mathrm{rpm}$ to $1000 \mathrm{rpm}(15 \mathrm{~s})$, the speed constant area which speed is equal to $1000 \mathrm{rpm}(2 \mathrm{~s})$ and the speed drop area which is from $1000 \mathrm{rpm}$ to $50 \mathrm{rpm}$.

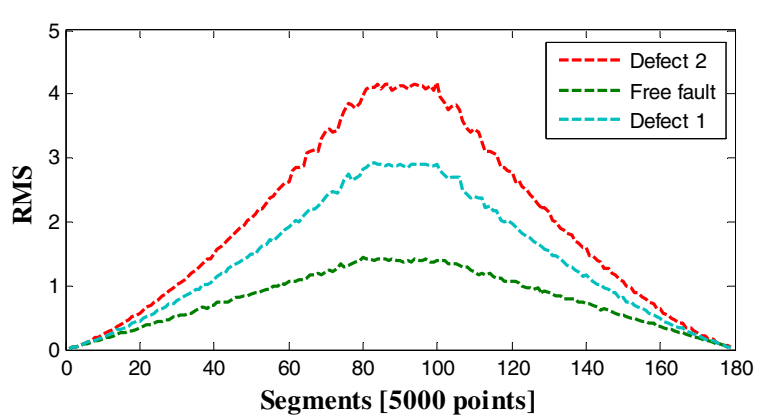

(a)

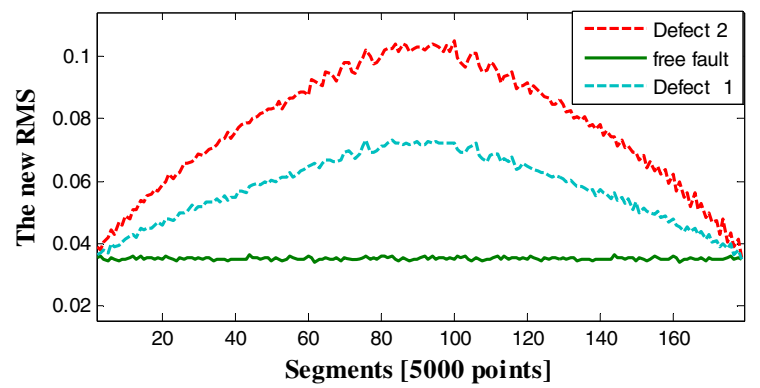

(b)

Fig. 4. (a) $R M S$ value for simulation signals for free fault and with fault (defect 1, defect 2), (b) $R M S$ value for simulation signals after division by instantaneous speed for free fault and with fault.

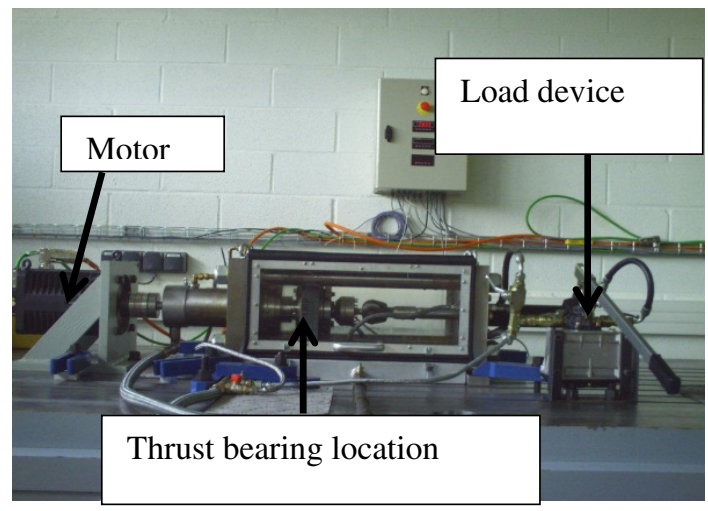

(a)

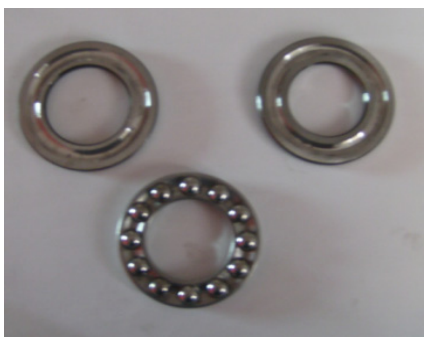

(b)

Fig. 5. (a) Test rigs (b) The used thrust bearing.

For four thrust bearings (see Fig. 5b) the spalling defects are created with different surfaces on one of its two rings. The surfaces of these defects are measured, and presented in Table 1. Figure 7 shows the microscopic photos for defects 1 and 4 . Using the loading device the following charges are applied: 0, 500, 1000, 1500, 2000, 2500 and 2900 daN to the thrust bearings for each degradation state. 


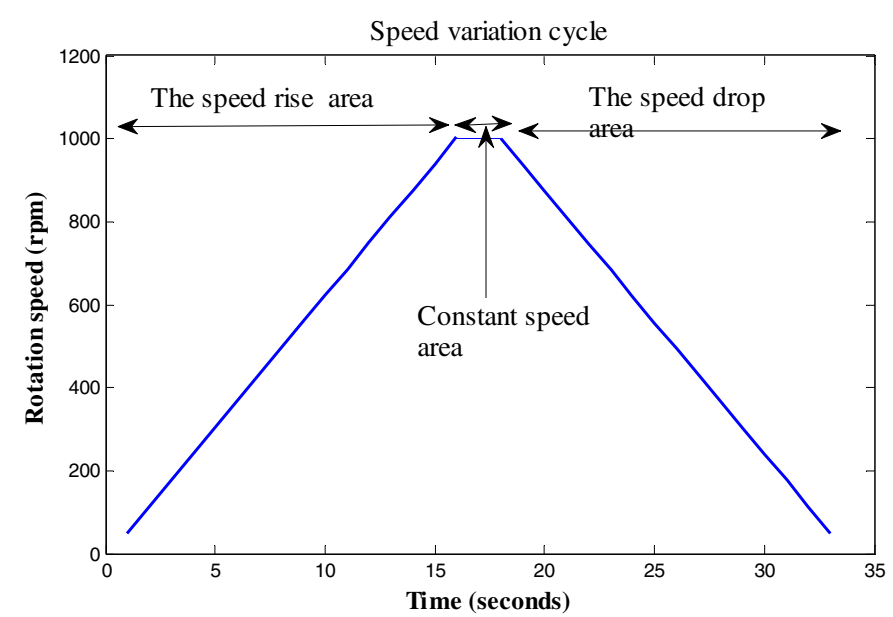

Fig. 6. Speed variation cycle.

Table 1. The created spalling defect surfaces $\left(\mathrm{mm}^{2}\right)$.

\begin{tabular}{ccccc}
\hline $\mathrm{N}^{\circ}$ of the defect & defect 1 & defect 2 & defect 3 & defect 4 \\
\hline Surface $\left(\mathrm{mm}^{2}\right)$ & 0.8 & 3.6 & 9.2 & 13.3 \\
\hline
\end{tabular}
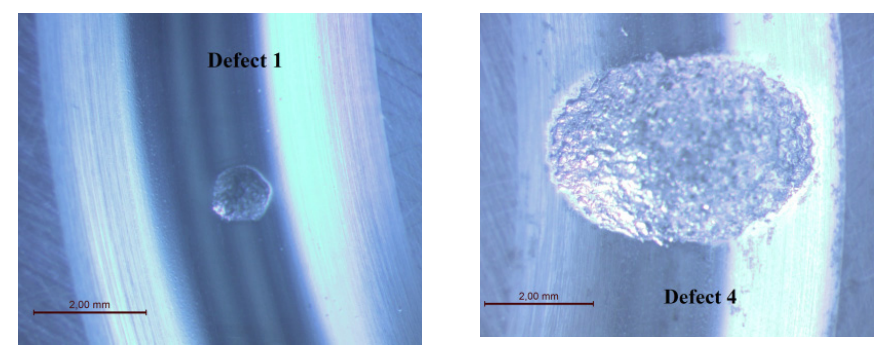

Fig. 7. Microscopic pictures of the spalling defect 1 and 4 .

For the instantaneous speed estimation, the signal from optical encoder is subdivided on slices (1024 points) and the Hilbert method is used on each slice as follow:

- Compute the discrete Fourier transform of each segment,

- Filter bandpass around the frequency of rotation of the shaft,

- Obtain the analytic signal and estimate the instantaneous phase,

- Estimate the instantaneous speed by deriving the instantaneous phase.

A smoothing is applied by replacing each point of segment by the average of the instantaneous speed estimated in this segment.

A subsampling by a factor of 4 is applied to the entire signal of the instantaneous speed estimated, to have the same size as the signal of the accelerometer sensor.

\section{Signal analysis}

Figure 8 shows the vibration signals acquired in the cases of free fault and defect 3 for the load 2900 daN. In the free fault case (Fig. 8a), we can observe the progressive rise in the vibration signal amplitude from $0.5 \mathrm{~m} . \mathrm{s}^{-2}$

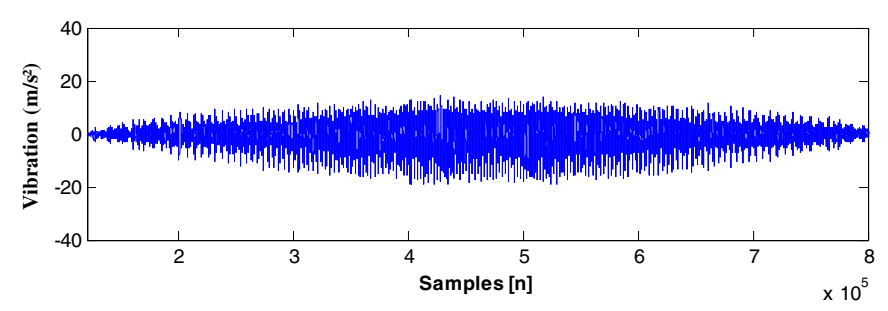

(a)

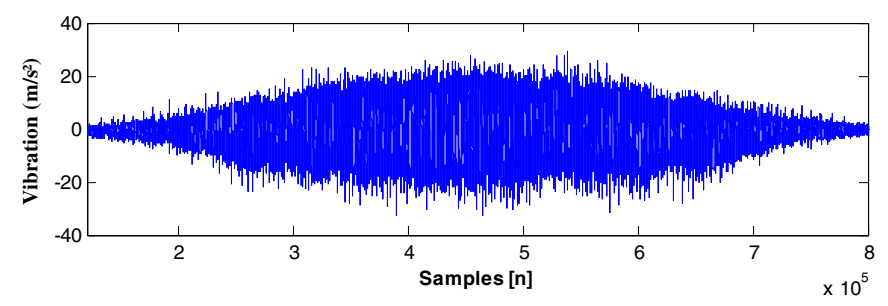

(b)

Fig. 8. (a) Typical signal recorded in the free fault case (load 2900 daN), (b) Typical signal recorded in the case of defect 3 (load 2900 daN).

to $14 \mathrm{~m} . \mathrm{s}^{-2}$ in the rising speed area and in the drop speed area the amplitude decreases from $14 \mathrm{~m} . \mathrm{s}^{-2}$ to $0.5 \mathrm{~m} . \mathrm{s}^{-2}$. It can be seen that the amplitude of the signal is modulated by the speed variation. In the case of defect 3 , the signal amplitude increases from $0.8 \mathrm{~m} . \mathrm{s}^{-2}$ to $30 \mathrm{~m} . \mathrm{s}^{-2}$ in the speed rise area and decreases from $30 \mathrm{~m} . \mathrm{s}^{-2}$ to $0.8 \mathrm{~m} . \mathrm{s}^{-2}$ in the speed drop area (see Fig. $8 \mathrm{~b}$ ). It is obvious that the vibration signal amplitude is more augmented compared to the free fault case due to the presence of defect. We can see that the vibration signal amplitude is modulated by the variation of the speed and the presence of spalling defect.

Subsequently, the signals acquired in the cases of the free fault and for the defects 1, 2, 3 and 4 are segmented in slices of 5000 points and on each slice the $R M S$ is calculated and is presented in Figure 9a. This figure shows that the $R M S$ values in the areas where speed rises and down follow a linear trend and these values depend strongly on the speed. The speed variation prevents us to put a fault detection threshold; for example, in this figure, if the $R M S$ value, equal to 3 , is taken as threshold, all the curves pass through this value.

To test the trend of $R M S$ values as a function of speed variation, Figure $9 \mathrm{~b}$ shows the linear regression of the points formed by the $R M S$ values in the speed rise area. To test the quality of the regression, the coefficients of determination $r^{2}$ are calculated: $r^{2}=0.9703$ in the free fault case, $r^{2}=0.9611$ in the case of defect 3 and $r^{2}=0.972$ in the case of defect 4 . We can see that these values are near to 1 which demonstrates the fact that the $R M S$ values of the vibratory signal are a linear function of the rotation speed.

Figure 10a shows the smoothed instantaneous speed estimated from the optical encoder. Its follows the same as in Figure 6, a rise time of 15 s (390000 samples), then the speed is stable for $2 \mathrm{~s}$ and then it decreases. 

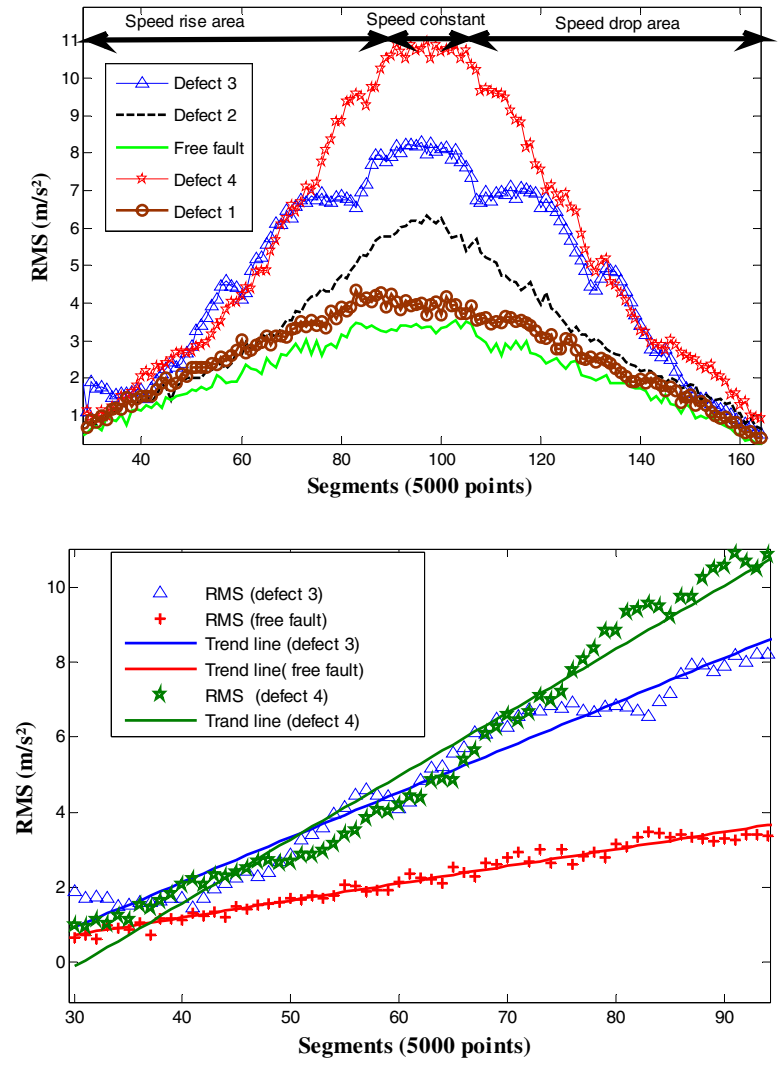

(b)

Fig. 9. (a) $R M S$ value depending on the thrust bearing states (b) Linear regression of the $R M S$ values for different states in the speed rise area.

Figure 10b presents the accelerometer signal from the fault 3 divided by the smoothed instantaneous speed. Compared to Figure 8b, the amplitude of the signal has a stationary trend. This figure clearly shows that dividing the signal vibration by the corresponding smoothed instantaneous speed eliminates the non-stationary appearance due to speed variation.

Figure 11 shows the different values of the new indicator $R M S$ for all states of the thrust bearing degradation for loads 1500 daN (Fig. 11a) and 2900 daN (Fig. 11b). It is found that the values of the new $R M S$ are quasistationary for each state. For each load, the $R M S$ values increase according to the state of the thrust bearing degradation. The more important is the defect the more the new $R M S$ values corresponding increase. It can be said that the new indicator values increase with the size of the defect regardless of the speed variation.

In the area corresponding to the speed rise (speed drop area is similar), the mean and standard deviation of the classical $R M S$ values (calculated for segments of 5000 points) are computed. We presented the mean value and standard deviation for the different states of degradation thrust bearings for load 1500 daN (Fig. 12a) and 2900 daN (Fig. 12b). It can be noted that in both cases the standard deviation is so large that if a threshold value is fixed, 3 for example, it can indicates all bearing degradation states. This is due to the presence of the slope which greatly influences this classical indicator.
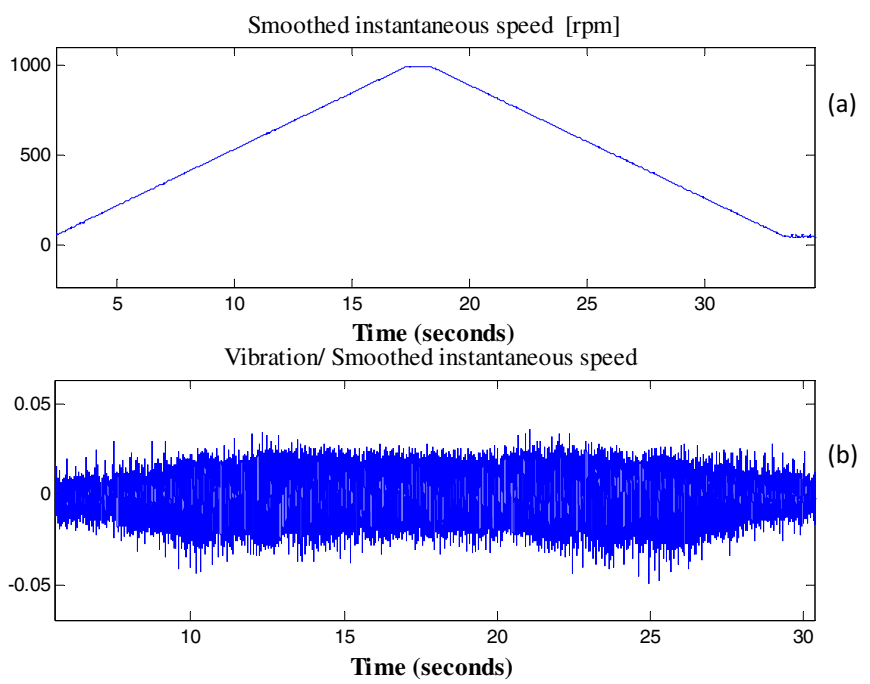

Fig. 10. (a) The smoothed instantaneous speed estimated from the optical encoder (b) Vibratory signal (Fig. 8b) divided by the smoothed instantaneous speed (defect 3 and load 2900 daN).

Figure 12c (respectively Fig. 12d) shows the mean value and standard deviation of the new $R M S$ (on slices of 5000 points) of the same vibration signals than in Figure 12a (respectively Fig. 12b), but after extraction of the instantaneous speed. It can be noted that the standard deviation around the mean became small in both load cases and it can be said that the new RMS indicator is a linear function of defects. From these figures, we can see that the new $R M S$ value increases with the surface defect. With this new indicator one can distinguish the case without defect and with spalling defect. This classification can be done from the surface of $2 \mathrm{~mm}^{2}$ for the load 1500 daN case and less than $2 \mathrm{~mm}^{2}$ in the case of load 2900 daN. This classification gives robustness to the new indicator in variable regime. For example if a threshold of value $25 \times(0.0001)$ is fixed in Figure 12c, one can be sure that at least the defect of surface $4 \mathrm{~mm}^{2}$ is present regardless of the change machine speed.

\section{Conclusion}

In this work, the issue of bearing elements monitoring in variable speed regime is treated. The speed variation generates non-stationary components in the vibration signals. Therefore it affects strongly the classical tools and prevents use these indicators for the diagnosis.

A model of the $R M S$ value according to the rotation speed, the load applied on the bearing, the defect size, the bearing geometry and the impulse response is established. Based on this model a new indicator is proposed; which is the $R M S$ value applied to the physical quantity vibration/instantaneous speed. This indicator is able to overcome the speed variation influence, which disrupts the classical $R M S$ indicator for bearings diagnosis.

A test on the simulate signals is performed thanks to a simple model of the vibration signal produced a single 

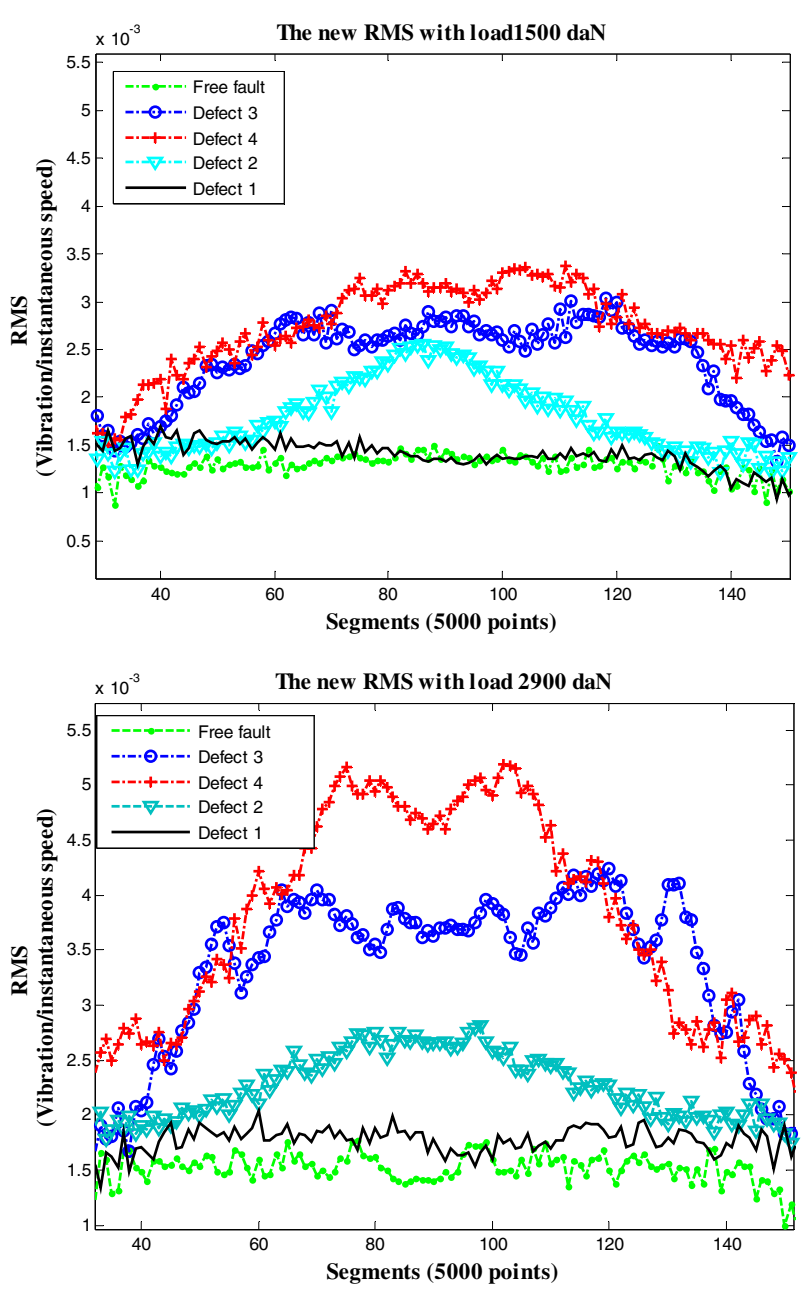

Fig. 11. The new $R M S$ value versus defects (a) load 1500 daN (b) load 2900 daN.

defect. The results of simulation clearly show that in the free fault case the new $R M S$ is constant whatever the speed variation and when defect begins the new $R M S$ rise depending on the defect severity.

A test rig was prepared to collect vibration signals and signals from the optical encoder, for different states of the thrust bearing, necessary to test the proposed method. It is found that the speed variation modulates the vibration signals. The results of applying the proposed method clearly show that the new $R M S$ value is independent of the speed variations influence. The new indicator is able to diagnose the bearing state whatever the speed variation and it can also classify the severity of the defect present in the rolling element bearings thing which is not achievable with classical $R M S$ value.

The proposed indicator requires only an optical encoder and an accelerometer sensor and is simple to implement in real time; in contrary to the other proposed methods like time frequency analysis [12] which needs information extraction methods in order to automatically identify the conditions of a machine in variable rotating speed regime.

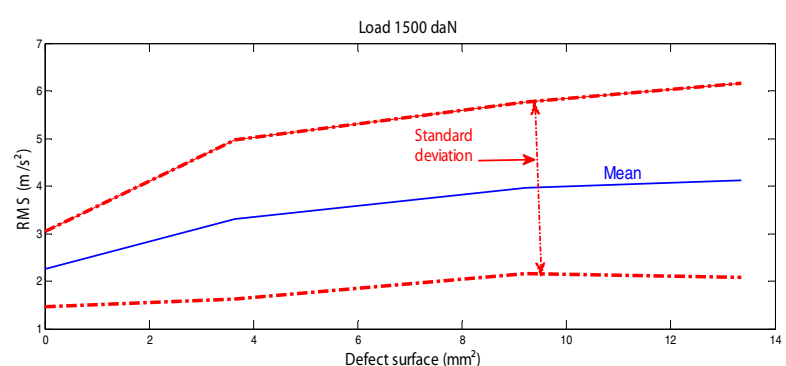

(a)

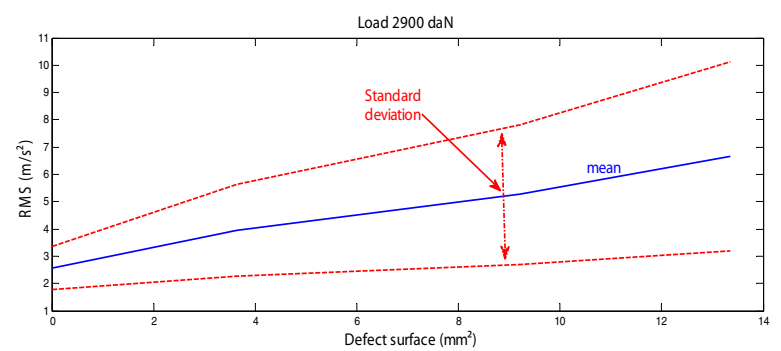

(b)

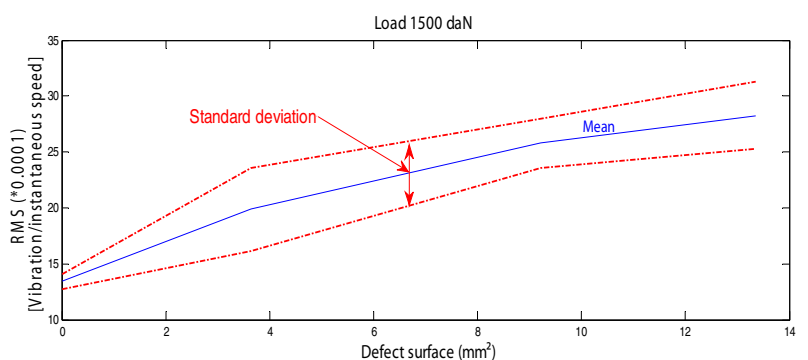

(c)

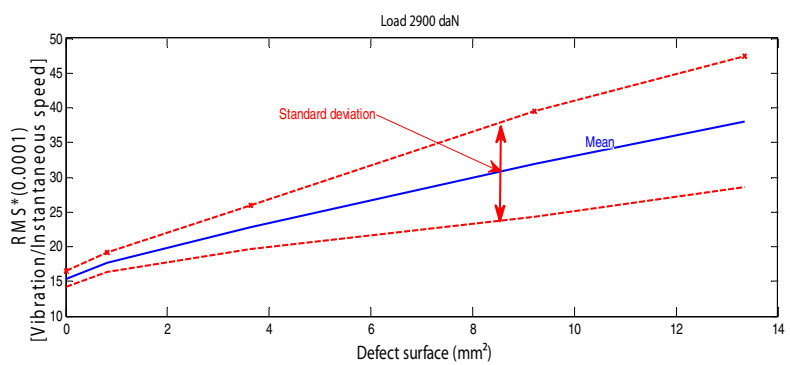

(d)

Fig. 12. Mean and dispersion of: (a) classical $R M S$ of the signal vibration - load 1500 daN, (b) classical $R M S$ of the signal vibration - load 2900 daN, (c) new $R M S$ value - load 1500 daN, (d) new $R M S$ value - load 2900 daN.

Acknowledgements. This paper was financially supported by the "Region of Champagne-Ardenne" and European's funds FEDER.

\section{References}

[1] B.T. Kuhnell, Wear in rolling element bearings and gears - how age and contamination affect them, Machinery Lubrication Magazine, Monash University, 2004

[2] N. Tandon, B.C. Nakra, Detection of defects in rolling element bearings by vibration monitoring, J. Institution of Engineers (India)- Mechanical Engineering Division (ISSN 0020-3408), 73 (1993) 271-282

[3] R.A Collacott, Mechanical fault diagnosis, Chapman and Hall, London, 1977 
[4] K.S. Jardine, D. Lin, D. Banjevic, A review on machinery diagnostics and prognostics implementing conditionbased maintenance, Mech. Syst. Signal Process. 20 (2006) $1483-1510$

[5] N. Tandon, A. Choudury, A review of vibration and acoustic measurement methods for the detection of defects in rolling element bearings, Tribol. Int. 32 (1999) 469-480

[6] PD. McFadden, J.D. Smith, Vibration monitoring of rolling element bearings by the high frequency resonance technique - a review. Tribol. Int. 17 (1984) 3-10

[7] PD. McFadden, J.D. Smith, Model for the vibration produced by a single point defect in a rolling element bearing, J. Sound Vibr. 96 (1984) 69-82

[8] PD. McFadden, J.D. Smith, The vibration produced by multiple point defects in a rolling element bearing, J. Sound Vibr. 98 (1985) 263-273

[9] Z.K. Zhu, R.Q. Yan, L.H. Luo, Z.H. Feng, F.R. Kong, Detection of signal transients based on wavelet and statistics for machine fault diagnosis, Mech. Syst. Signal Process. 23 (2009) 1076-1097

[10] R.B. Randall, J. Antoni, Rolling element bearing diagnostics-A tutorial, Mech. Syst. Signal Process. 25 (2011) 485-520

[11] J. Antoni, Cyclic spectral analysis in practice, Mech. Syst. Signal Process. 21 (2007) 597-630
[12] H. Wang, P. Chen, Fuzzy diagnosis method for rotating machinery in variable rotating Speed, IEEE Sensors J. 11 (2011) 23-34

[13] W. Bartelmus, R. Zimroz, A new feature for monitoring the condition of gearboxes in non-stationary operating conditions, Mech. Syst. Signal Process. 23 (2009) 15281534

[14] J. McBain, M. Timusk, Fault detection in variable speed machinery: Statistical parameterization, J. Sound Vibr. 237 (2009) 623-646

[15] N. Tandon, A. Choudury, An analytical model for the prediction of the vibration response of rolling element bearings due to localized defect, J. Sound Vibr. 205 (1997) 275-292

[16] C. Zhang, Defect detection and life prediction of rolling element bearings, Thesis, Georgia Institue of Technology, Chapt. V, pp. 97-123, 2001

[17] F. Bolaers, S. Rémond, X. Chiementin, S. Crequey, J.P. Dron, Modélisation de la force d'impact due à un écaillage de fatigue dans les roulements, Premier Colloque International IMPACT 2010, Djerba, Tunisie, 2010

[18] J. Antoni, R.B. Randall, Differential diagnosis of gear and bearing faults, ASME J. Vibr. Acoust. 124 (2002) 165-171

[19] J. Antoni, R.B. Randall, The spectral kurtosis: a useful tool for characterizing non-stationary signals. Mech. Syst. Signal Process. 20 (2006) 282-307 